\title{
The effect of omega-3 fatty acids supplementation on social and behavioral disorders of children with autism: a randomized clinical trial
}

Wpływ suplementacji kwasów tłuszczowych omega-3 na zaburzenia społeczne i behawioralne u dzieci z autyzmem: badanie kliniczne z randomizacją

\author{
1,2Saeid Doaei, ${ }^{3}$ Fatemeh Bourbour, ${ }^{4}$ Zohreh Teymoori, ${ }^{5}$ Faranak Jafari, ${ }^{6}$ Naser Kalantari, \\ ${ }^{7}$ Saheb Abbas Torki, ${ }^{3}$ Narges Ashoori, ${ }^{1}$ Shiva Nemat Gorgani, ${ }^{8}$ Maryam Gholamalizadeh
}

\begin{abstract}
${ }^{1}$ National Nutrition and Food Technology Research Institute, Faculty of Nutrition Sciences and Food Technology, Shahid Beheshti University of Medical Sciences, Tehran, Iran

${ }^{2}$ Research Center of Health and Environment, Guilan University of Medical Sciences, Rasht, Iran ${ }^{3}$ Department of Clinical Nutrition and Dietetics, Research Institute Shahid Beheshti University of Medical Science, Tehran, Iran

${ }^{4}$ Roudehen Islamic Azad University, Roudehen, Iran

${ }^{5}$ Department of Nursing, Faculty of Nursing and Midwifery, Kermanshah University of Medical Sciences, Kermanshah, Iran

${ }^{6}$ Department of Community Nutrition, School of Nutrition and Food Sciences, Shahid Beheshti University of Medical Sciences, Tehran, Iran

${ }^{7}$ Department of Nutrition, Faculty of Nutrition Sciences, Shiraz University of Medical Sciences, Shiraz, Iran ${ }^{8}$ Student Research Committee, Cancer Research Center, Shahid Beheshti University of Medical Sciences, Tehran, Iran
\end{abstract}

\begin{abstract}
Introduction: Some studies reported that essential fatty acids deficiencies can be related to autism spectrum disorders (ASD) in children. This study aimed to investigate the effect of omega-3 on social, verbal, and behavioral activities in ASD children.

Material and methods: A double-blind, randomized clinical trial study was conducted on 54 children with autism, who were assigned to the case $(n=28)$ and control $(n=26)$ groups. The cases received one capsule of 1000 mg omega- 3 daily and the controls received one capsule of $1000 \mathrm{mg}$ medium chain triglyceride daily as placebo for eight weeks. The Gilliam Autism Rating Scale-second edition (GARS-2) was used to assess the severity of autism and food frequency questionnaire (FFQ) was used to assess their dietary intake. All measurements were done at baseline and after the intervention.

Results: After adjusting for age, gender, birth weight, BMI, dietary intake, mother's age, and mother's BMI, the intervention group had significantly improved stereotyped behaviors $(p=0.02)$, social communication $(p=0.02)$, and the GARS score ( $p=0.001)$ after the intervention compared to the control group. No significant change was found in the score of social interaction subscale.

Conclusions: The findings indicate that omega-3 treatment improved autism characteristics including stereotyped behaviors and social communication. Further studies are needed to confirm these findings and to determine the underlying mechanisms.
\end{abstract}

Key words:

omega-3, autism, autism spectrum disorders, dietary fat. 


\section{Introduction}

Autism spectrum disorders (ASD) are neurological and developmental disabilities that affect social communication and behaviors, psychosocial competence and activities mainly in infants and children. Children with ASD are characteristic by disorder in neuronal connectivity, abnormal synaptic function, and neurotransmitter imbalances [1,2]. The prevalence of autism is higher in boys than girls and occurs in all regions, cultures, and socioeconomic classes [3]. In recent years, ASD has become highly prevalent and the prevalence of ASD is approximately 1 percent of the population in the United States [4]. A study in Iran reported that the ASD prevalence in school children was $1.9 \%$ [5]. Although the causes of autism remained unclear, environmental, genetic, and metabolic factors may play an important role in ASD [6]. Other factors involved in the development of autism include immunological, perinatal, neurodevelopmental, biochemical, psychosocial, and familial factors [7]. The treatment of autistic children needs to enhance their ability to integrate into school and make meaningful relationships with peers [7]. Some studies have reported that haloperidol and resperidone are effective in the treatment of autism but can cause side effects [8].

Initial evidence from clinical studies suggests that nutritional interventions may improve behavioral disorders in ADS [9-12]. Central nervous system contains poly unsaturated fatty acids (PUFA $_{\mathrm{s}}$ ) especially docosa-hexaenoic acid (DHA) and arachidonic acid which cannot be synthesized in the human body but must be provided by nutrition. PUFAs are essential for normal brain growth and function [12]. Dietary intake of n-3 polyunsaturated fatty acids including eicosa-pentaenoic acid (EPA) and $\mathrm{DHA}$, which are mainly found in fish and fish oil, may alter the risk of adult mental and neurological disorders [13, 14].

Some studies reported that fatty acid imbalances or deficiencies may be involved in childhood neurological disorders including attention deficit and hyperactivity disorders (ADHD), dyslexia, and the spectrum of autism disorders $[15,16]$. Given the increasing prevalence of autism and the lack of studies on the relationship between macronutrient intakes in autistic people, this study aimed to investigate the relationship between fatty acid intake and the status of autistic signs such as stereotyped behavior, social interactions, and social interaction in children with AS.

\section{Material and methods}

\section{Sample characteristics}

This study was done in form of a double-blind trial from March 2018 to January 2019 on 54 children with autism; this means that the patients and researchers were not aware of the arms of the study. Participants were randomly selected from Autism Charity Center in Tehran, Iran. The allocation to the groups was done through web-based randomization using https://www. randomizer.org. Sealed non-transparent envelopes with randomized sequences were used to hide the allocation. The diagnosis of ASD is done using diagnostic tools such as the Autism
Diagnostic Observation Scale (ADOS) and the DSM-IV TR criteria for a diagnosis of autistic disorder by an expert clinician [17].

\section{Inclusion and exclusion criteria}

Inclusion criteria included willingness to participate in the study, diagnosis of autism by a psychiatrist with moderate or severe autism symptoms, age from 5 to 15 years, and referring to the Autism Charity Center in Tehran. A drug-free interval of at least 6 weeks was required before baseline assessments were conducted. Sixty-four children with ASD were assessed for eligibility and met inclusion criteria. Sample size was calculated with $\alpha=0.95, \beta=20 \%$, ratio of unexposed to exposed of $2: 1$, and power of 0.8 . The exclusion criteria included the history of infection $(n=1)$, neurological disorders $(n=2)$, using any kind of medication $(n=1)$, history of allergy to fish or nuts $(n=1)$, consuming omega-3 supplementation past 2 months $(n=2)$, major medical illness $(n=1)$ and suffering from diabetes or blood disorders $(n=1)$, receiving a psychotropic drug effective on the behavior $(n=1)$. The final analysis was performed on fifty-four children with ASD who were assigned in the intervention $(n=28)$ and control $(n=26)$ groups. Parents of children complete a written informed consent. All participants, parents, and study personnel were blinded to group assignment and the randomized sequence list for the entire study, including the data analysis. All measurements were done at baseline and after eight weeks of the intervention

\section{Anthropometric measurements}

Height was measured using a measuring tape fastened to a wall and without shoes with a nearest $0.5 \mathrm{~cm}$. A bio impedance analysis scale (BIA) was used to measure body weight and body mass index (BMI). The extracted data was classified according to World Health Organization z-scores (for height, weight, and BMI).

\section{Dietary assessment}

A validated 168-item semi-quantitative food frequency questionnaire (FFQ) was completed through a face to face interview of the parents by a trained nutritionist in order to assessment the amount of fatty acids intake and estimation of every day food intake [18]. All data obtained from FFQ were converted to grams per day using Iranian household measures reference.

\section{Assessment of autism}

We used a validated form of the Psychometric Properties of Gilliam Autism Rating Scale (GARS) to assess Autistic Children [19]. The GARS scale is a standardized instrument for the assessment of autism and other severe behavioral disorders and contains four subscales and each subscale contains 14 items. The score for each question is between 0 and 3 [19]. The first subscale is stereotyped behaviors including stereotyped behaviors, motor disorders, and exotic behaviors. The second subscale is communication, includes items 15 to 28 which describe verbal and nonverbal behaviors that are signs of autism. Social interaction is the third subscale that includes items 29 to 42. The fourth subscale is developmental disorders, which includes items 43 to 56 on childhood developmental status. 


\section{Intervention}

This study was a randomized double blind placebo-controlled clinical trial. Eligible subjects were randomly divided into two groups. Group (1) received one capsule $1 \mathrm{gram}$ per day containing omega-3 Long Chain (180 mg EPA + 120 mg DHA) (Zahravi Company, Iran) and Group (2) received one capsule 1 gram per day containing medium chain triglyceride as placebo for eight weeks. For follow-up and monitoring, participants were contacted by telephone every week after randomization. The children were assessed at week eight for anthropometric assessments, the severity of autism assessment after intervention.

\section{Statistical analysis}

The comparison of qualitative variables between two groups was performed using chi-square test. Paired and independent $t$-test were used to detect any significant differences within and between two groups. Wilcoxon and Mann-Whitney nonparametric tests were applied to detect any significant differences within and between two groups respectively for variables that were not normally distributed. A value of $p<0.05$ was considered to be statistically significant. A mixed-model
ANOVA for repeated measures was used to identify the significant differences between two groups and between before and after intervention to eliminate the effects of confounders.

\section{Ethics Statement}

The study was approved by the Ethics Committee of the National Nutrition and Food Technology Research Institute, Tehran, Iran (reference number: Ir.sbmu.nnftri.rec. 054569, Approval date: 2014-09-23, 1393/07/01).

\section{Results}

A total of 54 children were included in the analysis, of which 28 were assigned to the intervention group and 26 were assigned to the control group (Fig. 1).

No significant differences were found between the groups at the baseline, except for sex (Table I). There were also no significant differences observed in anthropometric characteristics (children's and mother's height, weight, BMI and children's birth weight) between the groups at the baseline (Table I). Dietary intake of saturated fatty acids (SFAs; $p=0.04$ ) and mono un-

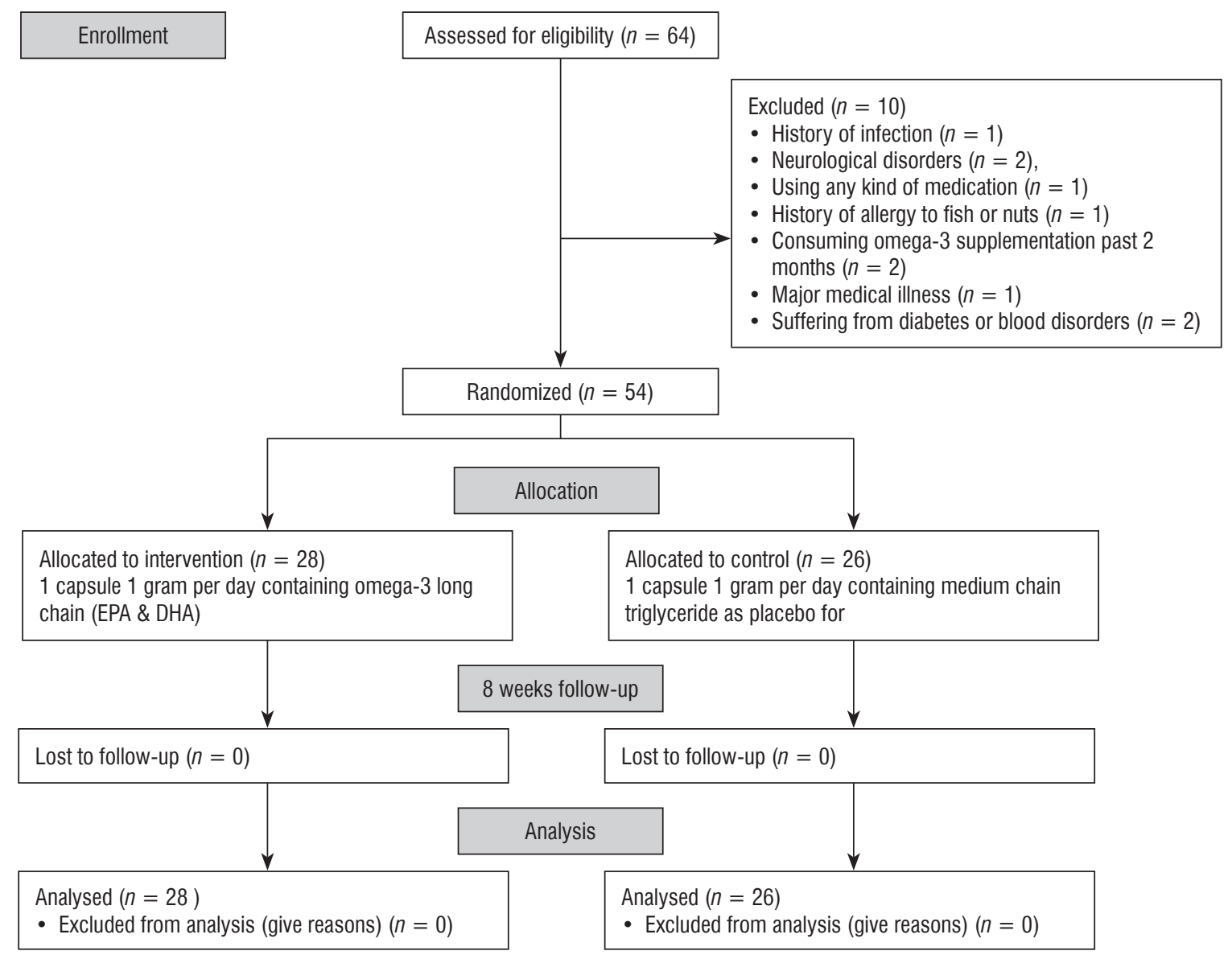

Figure 1. Study flowchart 
saturated fatty acids (MUFAs; $p=0.01$ ) were different between two groups and compared with the baseline, whereas poly unsaturated fatty acids (PUFAs; $p=0.07$ ) and the total fatty acids intake $(p=0.06)$ were not significantly different between two groups.

The intervention group had improved GARS score $(p=0.001)$, stereotyped behaviors $(p=0.02)$, and social communication $(p=0.02)$ after the intervention compared to the control group after adjusting for age, gender, birth weight, BMI, dietary fat intake, mother's age, and mother's BMI. No significant change was found in the status of social interaction (Table III).

\section{Discussion}

This study reported that after adjusting for age, gender, birth weight, BMI, dietary fat intake, mother's age, and mother's BMI, the intervention group had improved GARS score ( $p=0.001)$, stereotyped behaviors ( $p=0.02)$, and social communication

Table I. Baseline characteristics of the study participants [1, 2]

\begin{tabular}{|c|c|c|c|}
\hline \multirow[t]{2}{*}{ Variable } & \multicolumn{3}{|l|}{ Group } \\
\hline & Intervention group $(n=28)$ & Control group $(n=26)$ & $\mathrm{P}^{3}$ \\
\hline Age & $8.1 \pm 6.7$ & $8.2 \pm 3.6$ & 0.75 \\
\hline Mother's age & $36.8 \pm 0.9$ & $38.6 \pm 6.7$ & 0.23 \\
\hline \multicolumn{4}{|l|}{ Sex } \\
\hline Male & 19 & 20 & 0.00 \\
\hline Female & 9 & 6 & \\
\hline Height & $132.12 \pm 8.1$ & $133.16 \pm 6.3$ & 0.84 \\
\hline Weight & $33.14 \pm 9.1$ & $32.14 \pm 8.6$ & 0.66 \\
\hline BMl & $18.4 \pm 5.5$ & $17.3 \pm 7.9$ & 0.65 \\
\hline Birth weight & $2942.684 \pm 2.2$ & $2960.413 \pm 4.8$ & 0.97 \\
\hline Mother's height & $159.5 \pm 9$ & $161.5 \pm 6.7$ & 0.25 \\
\hline Mother's weight & $71.11 \pm 8.7$ & $69.9 \pm 3.8$ & 0.39 \\
\hline Mother's BMI & $28.4 \pm 1.7$ & $26.4 \pm 6.2$ & 0.19 \\
\hline
\end{tabular}

$\mathrm{BMl}$ - body mass index

Table II. Fatty acid intake between two groups

\begin{tabular}{llll}
\hline \multirow{2}{*}{ Variable } & Group & & \\
\cline { 2 - 4 } & Intervention group $(n=28)$ & Control group $(n=26)$ & $p$-value \\
\hline Fat (g/day) & $142.62 \pm 42$ & $109.33 \pm 66$ & 0.06 \\
\hline Saturated fatty acids (g/day) & $48.48 \pm 13$ & $29.11 \pm 9.6$ & 0.04 \\
\hline MUFA (g/day) & $52.19 \pm 19$ & $39.12 \pm 6.6$ & 0.01 \\
\hline PUFA (g/day) & $35.16 \pm 10$ & $27.9 \pm 10.1$ & 0.07 \\
\hline
\end{tabular}

Data is expressed as mean \pm standard deviation for all variables 
Table III. Effect of omega-3 supplementations on autism according to scores of GARS and its subscales using repeated measures $(n=54 ; \mathrm{df}=1)$

\begin{tabular}{|c|c|c|c|c|c|c|}
\hline \multirow[t]{2}{*}{ Variable } & \multicolumn{2}{|c|}{ The intervention group $(n=28)$} & \multicolumn{2}{|c|}{ The control group $(n=26)$} & \multicolumn{2}{|c|}{ Group $\times$ time } \\
\hline & At baseline & After 8 weeks & At baseline & After 8 weeks & $\mathrm{F}$ & $p$-value \\
\hline Stereotyped Behaviors & $20.39 \pm 5.07$ & $19.07 \pm 5.06$ & $21.73 \pm 6.59$ & $21.92 \pm 6.47$ & 5.926 & 0.02 \\
\hline Social Communication & $22.7857 \pm 5.34$ & $20.50 \pm 5.09$ & $24.04 \pm 7.16$ & $24.42 \pm 6.87$ & 5.44 & 0.02 \\
\hline Social Interaction & $21.03 \pm 4.25$ & $20.11 \pm 3.98$ & $22.84 \pm 4.36$ & $22.61 \pm 4.08$ & 1.81 & 0.18 \\
\hline GARS-2 & $64.21 \pm 10.51$ & $59.58 \pm 10.62$ & $68.61 \pm 12.86$ & $68.96 \pm 13.24$ & 13.14 & 0.001 \\
\hline
\end{tabular}

Adjusted for age, gender, birth weight, BMI, dietary fat intake, mother's age, and mother's BMI

$(p=0.02)$ after the intervention compared to the control group. No significant change was found in the social interaction subscale.

In line with this study, Amminger et al. investigated the omega-3 fatty acid supplementation in autistic children and found that omega-3 treatment reduced hyperactivity and stereotyped behavior [20]. Another study also reported that omega-3 supplementation for 12 weeks decreased hyperactivity in ASD children [21]. Lyall et al. investigated the association between maternal dietary fat intake before or during pregnancy and ASD in children. They reported that maternal linoleic acid intake was significantly associated with the risk of ASD [22].

Ansary et al. reported that the plasma fatty acid concentrations were changed in autism patients, especially increased in saturated fatty acids except propionic acid and decreased in PUFA [23]. The omega-3 and omega-6 supplementation for 3 months improved language development in children at risk for ASD [24]. A systematic review identified that omega-3 supplementation improved attention deficiency and hyperactivity in children with autism [25]. Another study reported that omega-3 treatment improved reading skills in children with lowest initial reading skills [26]. Omega-3 treatment may also have some influences on improvement of depression and schizophrenia $[27,28]$. Richardson et al. reported that EPA (3 g) or fish oil $(10 \mathrm{~g} / \mathrm{d})$ improved symptoms in schizophrenia spectrum disorders, depression, attention deficiency, hyperactivity disorder (ADHD), dyslexia, and dyspraxia disorder [29]. Another study reported that $\mathrm{DHA}$ and omega-3 concentration significantly decreased in ASD children [16]. The findings of one placebocontrolled trial reported that omega-3 treatment could improve behavioral measures, reading and spelling capacities and reduce impulsivity in DCD (Developmental Coordination Disorder) children [29-31]. These findings suggested that omega-3 treatment could be effective for treating aggression and impulsivity [29-31]. In contrast with current study, Bent et al. reported that omega-3 supplementation did not affect hyperactivity in children with ASD. This is probably due to small sample size
(27 autistic chidren in total) and the mild level of hyperactivity in both the placebo and omega-3 treatment groups [21]. The underlying mechanism of the effects of omega-3 on brain and ASD symptoms is not yet understood, but may be associated with modulation of serotonergic and dopaminergic systems. The DHA or the DHA-ARA ratio could be associated with noradrenergic system [32, 33]. EPA plus DHA supplementation lowered plasma NE concentrations in normal volunteers even at the small dose of $762 \mathrm{mg}$ of EPA plus DHA per day. DHA may also change the central noradrenergic system that plays a key role in modifying impulsive behaviors related to these neurotransmitters such as aggression [32, 33]. Moreover, DHA and EPA have key roles in production of membrane phospholipids, especially in the central nervous system [34]. The macronutrients including omega-3 fatty acids are supposed to have neuroprotective role in production of the synaptic maintenance [35], modulation of brain cell signaling, regulation of monoamines production, and receptor signal transduction pathway [36-38], which could explain the role of omega-3 in psychiatric diseases such as autism [38-39]. Omega-3 PUFAs and their metabolic products provide a solid foundation because they play a role in ASD through their role in brain structure and brain function, neurotransmission, cell membrane structure, and microbial domain organization [40]. DHA is highly enriched in neural and synaptic membranes, indicating an important role in neuronal cell signaling. It is preferentially incorporated into phosphatidylethanolamine and phosphatidylserine in the inner layer of synapse membranes, and its sterile incompatibility with cholesterol causes either DHA- or cholesterol-rich lipid rafts. DHA also affects membrane fatty acid chain fluidity, ion permeability, elasticity, protein function, phase behavior, and fusion [41].

This study had some limitations. Although the sample size was calculated with the power accepted for the study, the results should be confirmed in larger studies. Further studies are needed to increase our understanding of the effect of omega-3 fatty acids supplementation on social and behavioral disorders of children with autism. 


\section{Conclusions}

This study found that omega-3 supplementation improved GARS score, stereotyped behaviors, and social communica-

\section{References}

1. Pardo CA, Eberhart CG. The neurobiology of autism. Brain Pathol 2007; 17: 434-447. doi: 10.1111/j.1750-3639.2007.00102.x

2. Belmonte MK, Allen G, Beckel-Mitchener A, et al. Autism and abnormal development of brain connectivity. J Neurosci 2004; 24 : 9228-9231. doi: 10.1523/JNEUROSCI.3340-04.2004

3. Matson JL, Kozlowski AM. The increasing prevalence of autism spectrum disorders. Res Autism Spectrum Disord 2011; 5: 418-425.

4. Ghanizadeh A. A preliminary study on screening prevalence of pervasive developmental disorder in schoolchildren in Iran. J Autism Dev Disord 2008; 38: 759-763. doi: 10.1007/s10803-007-0445-6

5. Kumar RA, Christian SL. Genetics of autism spectrum disorders. Curr Neurol Neurosci Rep 2009; 9: 188-197. doi: 10.1007/s11910009-0029-2

6. Posey DJ, McDougle CJ. Pharmacotherapeutic management of autism. Expert Opin Pharmacother 2001; 2: 587-600.

7. Sadock BJ, Sadock VA. Synopsis of psychiatry behavioral sciences, $10^{\text {th }}$ ed. Translated by Rezaei F. Arjmand Press, Tehran 2007; 3: 446-455.

8. Chez MG, Burton Q, Dowling T, et al. Memantine as adjunctive therapy in children diagnosed with autistic spectrum disorders: an observation of initial clinical response and maintenance tolerability. J Child Neurol 2007; 22: 574e579. doi: 10.1177/0883073807302611

9. Dosman CF, Brian JA, Drmic IE, et al. Children with autism: effect of iron supplementation on sleep and ferritin. Pediatr Neurol 2007; 36 : 152e158. doi: 10.1016/j.pediatrneurol.2006.11.004

10. Miral S, Gencer O, Inal-Emiroglu FN, et al. Risperidone versus haloperidol in children and adolescents with AD: a randomized, controlled, double-blind trial. Eur Child Adolesc Psychiatry 2008; 17: 1-8. doi: 10.1007/s00787-007-0620-5

11. Doaei S, Hajiesmaeil M, Aminifard A, et al. Effects of gene polymorphisms of metabolic enzymes on the association between red and processed meat consumption and the development of colon cancer; a literature review. J Nutr Sci 2018; 7: e26. doi: 10.1017/ jns.2018.17

12. Wainwright PE. Dietary essential fatty acids and brain function: a developmental perspective on mechanisms. Proc Nutr Soc 2002; 61: 61-69. doi: 10.1079/pns2001130

13. Berger GE, Wood SJ, Pantelis C, et al. Implications of lipid biology for the pathogenesis of schizophrenia. Aust N Z J Psychiatry 2002; 36: 355-366. doi: 10.1046/j.1440-1614.2001.01021.x

14. Young G, Conquer J. Omega-3 fatty acids and neuropsychiatric disorders. Reprod Nutr Dev 2005; 45: 1-28. doi: 10.1051/rnd:2005001

15. Richardson AJ. Long-chain polyunsaturated fatty acids in childhood developmental and psychiatric disorders. Lipids 2004; 39 : 1215-1222. doi: 10.1007/s11745-004-1350-z

16. Vancassel S, Durand G, Barthelemy $C$, et al Plasma fatty acid levels in autistic children. Prostaglandins Leukot Essent Fatty Acids 2001; 65: 1-7. doi: 10.1054/plef.2001.0281 tion variables in the intervention group compared with the control group. Further studies with larger sample sizes and longer period of the intervention are needed to clarify the efficacy of omega-3 supplementation on ASD children.

17. American Psychiatric Association. Diagnostic and Statistical Manual of Mental Disorders, $4^{\text {th }}$ ed. Washington, DC: American Psychiatric Press, 1994.

18. Esmaillzadeh A, Kimiagar M, Mehrabi Y, et al. Dietary patterns, insulin resistance, and prevalence of the metabolic syndrome in women. Am J Clin Nutr 2007; 85: 910-918. doi: 10.1093/ajcn/85.3.910

19. Ahmadi SJ, Safari T, Hemmatiyan M, et al. The Psychometric Properties of Gilliam Autism Rating Scale (GARS). Uijs 2011; 1: 87-104.

20. Amminger GP, Berger GE, Schäfer MR, et al. Omega-3 fatty acids supplementation in children with autism: a double-blind randomized, placebo-controlled pilot study. Biol Psychiatry 2007; 61: 551-553. doi: 10.1016/j.biopsych.2006.05.0072

21. Bent S, Bertoglio K, Ashwood P, et al. A pilot randomized controlled trial of omega-3 fatty acids for autism spectrum disorder. J Autism Dev Disord 2011; 41: 545-554. doi: 10.1007/s10803-010-1078-8

22. Lyall K, Munger KL, O'Reilly ÉJ, et al. Maternal dietary fat intake in association with autism spectrum disorders. Am J Epidemiol 2013; 178: 209-220. doi: 10.1093/aje/kws433

23. El-Ansary AK, Bacha AG, Al-Ayahdi LY. Plasma fatty acids as diagnostic markers in autistic patients from Saudi Arabia. Lipids Health Dis 2011; 10: 62. doi: 10.1186/1476-511X-10-62

24. Lachman A. New developments in diagnosis and treatment update: Schizophrenia/first episode psychosis in children and adolescents. J Child Adolesc Ment Health 2014; 26: 109-124. doi: 10.2989/17280583.2014.924416

25. Bloch MH, Qawasmi A. Omega-3 fatty acid supplementation for the treatment of children with attention-deficit/hyperactivity disorder symptomatology: systematic review and meta-analysis. J Am Acad Child Adolesc Psychiatry 2011; 50: 991-1000. doi: 10.1016/j. jaac.2011.06.008

26. Richardson AJ, Burton JR, Sewell RP, et al. Docosahexaenoic acid for reading, cognition and behavior in children aged 7-9 years: a randomized, controlled trial (the DOLAB Study). PloS One 2012; 7: e43909.

27. Amminger GP, Schafer MR, Papageorgiou K, et al. Long-chain omega-3 fatty acids for indicated prevention of psychotic disorders: a randomized, placebo-controlled trial. Arch Gen Psychiatry 2010; 67: 146-154. doi: 10.1001/archgenpsychiatry.2009.192

28. Freeman MP, Hibbeln JR, Wisner KL, et al. Omega-3 fatty acids: evidence basis for treatment and future research in psychiatry. J Clin Psychiatry 2006; 67: 1954-1667. doi: 10.4088/jcp.v67n1217

29. Richardson AJ, Montgomery P. The Oxford-Durham study: a randomized, controlled trial of dietary supplementation with fatty acids in children with developmental coordination disorder. Pediatrics 2005; 115: 1360-1366. doi: 10.1542/peds.2004-2164

30. Yehuda S, Rabinovitz S, Carasso RL, et al. The role of polyunsaturated fatty acids in restoring the aging neuronal membrane. Neurobiol Aging 2002; 23: 843-853. doi: 10.1016/s0197-4580(02)00074-x

31. Zanarini MC, Frankenburg FR. Omega-3 fatty acid treatment of women with borderline personality disorder: a double-blind, 
Doaei S., Bourbour F., Teymoori Z., Jafari F., Kalantari N., Torki S.A., Ashoori N., Gorgani S.N., Gholamalizadeh M.

placebo-controlled pilot study. Am J Psychiatry 2003; 160: 167169. doi: 10.1176/appi.ajp.160.1.167

32. Hibbeln JR, Linnoila M, Umhau JC, et al. Essential fatty acids predict metabolites of serotonin and dopamine in cerebrospinal fluid among healthy control subjects, and early-and late-onset alcoholics. Biol Psychiatry 1998; 44: 235-242. doi: 10.1016/s00063223(98)00141-3

33. Hamazaki K, Itomura M, Huan M, et al. Effect of $\omega$-3 fatty acid-containing phospholipids on blood catecholamine concentrations in healthy volunteers: a randomized, placebo-controlled, double-blind trial. Nutrition 2005; 21: 705-710. doi: 10.1016/j.nut.2004.07.020

34. Lauritzen L, Brambilla P, Mazzocchi, A, et al. DHA Effects in Brain Development and Function. Nutrients 2016; 8: 6. doi: 10.3390/ nu8010006

35. Mischoulon D. Freeman MP. Omega-3 fatty acids in psychiatry. Psychiatr Clin North Am 2013; 36: 15-23. doi: 10.1016/j.psc.2012.12.002

36. Ross BM, Seguin J, Sieswerda LE. Omega-3 fatty acids as treatments for mental illness: Which disorder and which fatty acid? Lipids Health Dis 2007; 6: 21. doi: 10.1186/1476-511X-6-21
37. Hallahan B, Garland MR. Essential fatty acids and mental health. $\mathrm{Br}$ J Psychiatry 2005; 186: 275-277. doi: 10.1192/bjp.186.4.275

38. Doaei S, Kalantari N, Izadi P, et al. Interactions between macronutrients' intake, FTO and IRX3 gene expression, and FTO genotype in obese and overweight male adolescents. Adipocyte 2019; 8: 386-391. doi: 10.1080/21623945.2019.1693745

39. Galli C, Trzeciak HI, Paoletti R. Effects of essential fatty acid deficiency on myelin and various subcellular structures in rat brain. J Neurochem 1972; 19: 1863-1867. doi: 10.1111/j.1471-4159.1972. tb01475.x

40. Agostoni C, Nobile M, Ciappolino V, et al. The role of omega-3 fatty acids in developmental psychopathology: a systematic review on early psychosis, autism, and ADHD. Int J Mol Sci 2017; 18: 2608. doi: 10.3390/ijms18122608.

41. Healy-Stoffel M, Levant B. N-3 (omega-3) fatty acids: Effects on brain dopamine systems and potential role in the etiology and treatment of neuropsychiatric disorders. CNS Neurol Disord Drug Targets 2018; 17: 216-232. doi: 10.2174/18715273176661804121 53612 